\title{
Distribution of the magnetic field and current density in superconducting films of finite thickness
}

\author{
D. Yu. Vodolazov, I.L.Maksimov *† \\ Nizhny Novgorod University, 603600, Russia.
}

February 1, 2008

\begin{abstract}
A one-dimensional equation describing the distribution of the effective vector potential $\bar{A}(y)$ across a film width, which holds for thin $(d<\lambda)$ and thick $(d>\lambda)$ films alike, is derived based on the analysis of a $2 \mathrm{D}$ Maxwell-Londons equation for superconducting films in a perpendicular magnetic field. The validity of this equation for a finite-thickness film is verified by a numerical analysis. An approximation dependence $\bar{A}(y)$, finite (with all of its derivatives) across the entire film width, is found for films, being in the Meissner state. The flux-entry field is evaluated for a film of arbitrary thickness. An approximation expression is obtained for the distribution of the sheet current density in the mixed state of a pin-free superconducting film with an edge barrier. The latter approximation allows to estimate magnetic field concentration factor at the film edge as a function of external magnetic field and geometrical parameters of the sample.
\end{abstract}

PACS: 74.60.Ec; 74.76.-w

Keywords: Meissner state; Mixed state; Superconducting films; Surface barrier

\section{Introduction}

Of active interest recently is theoretical [1, 2, 3, 4, 5, 6, 7, 8, 9] and experimental [9, 10 investigation of mixed static and dynamic states in superconducting films in a perpendicular magnetic field. Theoretical calculations of various magnetic characteristics of films in such a geometry, using the microscopic theory or the Ginzburg-Landau equations does not seem possible because of the mathematical intricacies involved in their solution.Therefore, in practical calculations the following two approaches are mostly employed. In the first method the Maxwell equation is analysed jointly with the London equation, which yields an integral-differential equation [1, 4, 5, 6, 7, 8] for distribution of the current density integrated over the film thickness $d$. This equation was derived on the assumption that the film is thin $(d \ll \lambda, \lambda$ is the London penetration depth), which naturally limits its applicability range.

The other approach is based on the theory of complex variable functions (TCVF) [3, 9], used in transformation of integral equations [2]. In this case the phenomenological dependences $\mathbf{B}(\mathbf{H})$ and $\mathbf{J}(\mathbf{E})$ are employed as an additional condition instead of the London equation. It should be noted that neither of these methods has ever focussed on investigating the effects related to finiteness of the London penetration depth $\lambda$. Besides, the equality of the results obtained through solving of the integral equation for thin films and by the use of the TCVF methods for thick films leads us to believe that for films of arbitrary thickness there should exist one equation describing distribution of the current density and the magnetic field across the film width.

The present paper deals with a study on the distribution of the magnetic field and current density in the Meissner and mixed states for films placed in a perpendicular magnetic field. It is shown that for the finite-

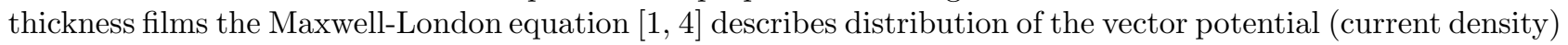
averaged over the film thickness $d$, provided the latter, is much smaller than the film width $W: d / W \ll 1$. For thin films, $d \ll \lambda$, this equation is valid practically over the entire width of the film, while in the case $d \gg \lambda$ it holds everywhere in the film, except for the areas near the edges, which measure as $W / 2<|Y| \leq W / 2-d / 2$.

\footnotetext{
${ }^{*}$ Corresponding author. Tel.: +7-831-2656255; Fax: +7-831-2658592; E-mail: ilmaks@phys.unn.runnet.ru.

$\dagger$ Current address. 23 Gagarin ave., Nizhny Novgorod University, Nizhny Novgorod, 603600, Russia.
} 
The paper is organized as follows. Section 2 describes derivation of an one-dimensional equaton for distribution of the film-thickness-averaged vector potential across a sample width, based on the analysis of 2D Maxwell-London equation. In subsections 2.1 and 2.2 the 2D and 1D equations are numerically studied and compared for thin and thick films respectively. An approximation dependence for $\bar{A}(y)$ is obtained through numerical solution of these equations. The distribution of the vector potential (or the local current density) and of the local magneti field over a superconductor cross-section is described. Section 3 deals with estimation of the field for the first vortex entry into thin and thick superconducting films (subsection 3.1 and 3.2 respectivly). In subsection 3.3 we discuss the influence of surface defects and a layered structure of superconductors on a barrier suppression field value. Section 4 is concerned with the structure of a mixed state in thin and thick superconducting films of an arbitrary width in the absence of bulk pinning. An approximation formula is found for the integral (over thickness) current density, which is then used as a basis for constructing the magnetization curves for these superconductors. Section 5 sums up the main results obtained in this work.

\section{The structure of the Meissner state}

Assume an infinite (in the $X$ direction) superconducting film of width $W$ and thickness $d$ in a perpendicular magnetic field; the geometry is shown in Fig.1. Let us first consider the Meissner state. The Maxwell equation has the form (in a gauge $\nabla \cdot \mathbf{A}=0$ )

$$
\Delta \mathbf{A}=-\frac{4 \pi}{c} \mathbf{j}
$$

where $\Delta$ is the $2 \mathrm{D}$ Laplacian operator. It also follows from the symmetry of the problem that only the $x$ components of the vector potential $\mathbf{A}=\left(A_{x}, 0,0\right)$ and of the current density $\mathbf{j}=\left(j_{x}, 0,0\right)$ are not zero. The boundary conditions are $\left.\frac{\partial A_{x}}{\partial Y}\right|_{Y \rightarrow \pm \infty}=-H_{\infty},\left.\frac{\partial A_{x}}{\partial Z}\right|_{Z \rightarrow \pm \infty}=0$, where $H_{\infty}$ is the field far from film $\mathbf{H}=\left(0,0, H_{\infty}\right)$. It should be noted that by the magnetic field $H$ in a film here we imply a microscopic field averaged over scales much larger than the atomic one but much smaller than $\lambda$.

In this case it is convenient to change over from the differential equation (1) to its integral analog. Using the Green function of the 2D Laplacian operator, we rewrite Eq.(1) as

$$
A_{x}(Y, Z)=A_{x}^{0}(Y)-\frac{2}{c} \int_{-W / 2}^{W / 2} \int_{-d / 2}^{d / 2}\left(\ln \left|\mathbf{R}-\mathbf{R}^{\prime}\right|+C\right) j_{x}\left(Y^{\prime}, Z^{\prime}\right) d Y^{\prime} d Z^{\prime},
$$

where $C$ is the constant generic for the $2 \mathrm{D}$ Green function, $A_{x}^{0}(Y)$ is the vector potential of an external field: $A_{x}^{0}(Y)=-H_{\infty} Y$ and the origin of coordinates is chosen in the film centre.

Employing London equation $\mathbf{j}=-\mathbf{A} / 4 \pi \lambda^{2}$ and introducing dimensionless coordinates $y=2 Y / W, z=2 Z / d$, Eq.(2) reads

$$
\begin{gathered}
A_{x}(y, z)=A_{x}^{0}(y)+\frac{W d}{16 \pi \lambda^{2}} \int_{-1}^{1} \int_{-1}^{1} \ln \left(\left(y-y^{\prime}\right)^{2}+\left(\frac{d}{W}\right)^{2}\left(z-z^{\prime}\right)^{2}\right) A_{x}\left(y^{\prime}, z^{\prime}\right) d y^{\prime} d z^{\prime} \\
+\frac{W d}{16 \pi \lambda^{2}} \tilde{C} \int_{-1}^{1} \int_{-1}^{1} A_{x}\left(y^{\prime}, z^{\prime}\right) d y^{\prime} d z^{\prime}
\end{gathered}
$$

where $\tilde{C}=C+2 \ln (W / 2)$. The latter integral in (3) is directly proportional to the total current. In a magnetic field (without a transport current) the total current is equal to zero due to the symmetry, so the last term in the right-hand side of Eq. (3) vanishes. We now average Eq.(3) over the film thickness, which yields the following expression (hereafter by $\bar{A}(y)$ we imply $0.5 \int_{-1}^{1} A_{x}(y, z) d z$ )

$$
\begin{gathered}
\bar{A}(y)=-H_{\infty} y W / 2+\frac{W d}{4 \pi \lambda^{2}} \int_{-1}^{1} \ln \left|y-y^{\prime}\right| \bar{A}\left(y^{\prime}\right) d y^{\prime} \\
+\frac{W d}{32 \pi \lambda^{2}} \int_{-1}^{1} \int_{-1}^{1} \int_{-1}^{1} \ln \left(1+b^{2} /\left(y-y^{\prime}\right)^{2}\right) A_{x}\left(y^{\prime}, z^{\prime}\right) d y^{\prime} d z d z^{\prime}
\end{gathered}
$$

where the integral kernel of Eq.(3) is written in the form 


$$
\ln \left(\left(y-y^{\prime}\right)^{2}+\left(\frac{d}{W}\right)^{2}\left(z-z^{\prime}\right)^{2}\right)=2 \ln \left|y-y^{\prime}\right|+\ln \left(1+\frac{b^{2}}{\left(y-y^{\prime}\right)^{2}}\right)
$$

and the designation $b=\left(z-z^{\prime}\right) d / W \ll 1$ is introduced (as in this case of superconducting films $d / W \ll 1$ ).

The function $\ln \left(1+b^{2} /\left(y-y^{\prime}\right)^{2}\right)$ is not zero only in a small region around point $y^{\prime}:\left|y-y^{\prime}\right| \leq|b|$. In this case integration over $y^{\prime}$ in the second integral of Eq.(4) can be done only over this small region. For the same reason we can expand the function $A_{x}\left(y^{\prime}, z^{\prime}\right)$ into the Taylor series in terms of $y^{\prime}$ near point $y$ :

$$
A_{x}\left(y^{\prime}, z^{\prime}\right)=A_{x}\left(y, z^{\prime}\right)+\frac{\partial A_{x}\left(y, z^{\prime}\right)}{\partial y}\left(y^{\prime}-y\right)+.
$$

Note that expansion (5) (in the above specified limit) is valid for thin $(d<\lambda)$ films over the entire sample width. In thick $(d>\lambda)$ films the validity of this expansion is violated in the near-edge regions with dimensions of order $d / W$. More details on the applicability of Eq.(5) are provided in the end of this Section.

After the series-expansion of function $A_{x}\left(y^{\prime}, z^{\prime}\right)(5)$ it is now possible to calculate the last term of Eq.(4)

$$
\int_{y-b}^{y+b} \int_{-1}^{1} \int_{-1}^{1} \ln \left(1+\left(\frac{d}{W}\right)^{2}\left(z-z^{\prime}\right)^{2} /\left(y-y^{\prime}\right)^{2}\right)\left(A_{x}\left(y, z^{\prime}\right)+\frac{\partial A_{x}\left(y, z^{\prime}\right)}{\partial y}\left(y^{\prime}-y\right)+\ldots\right) d y^{\prime} d z d z^{\prime}
$$

(note, that the upper (lower) limit of the integration over $y^{\prime}$ will change by $1(-1)$, when point $y$ becomes close to the film edge, i.e., when $1-|b| \leq||<1$; this takes place because the integration in (4) is carried out only over a sample volume) and to show that (6) is equal to zero in a wide parameters range. Indeed, integration of Eq.(6), first in terms of $y^{\prime}$ and then in $z$ and $z^{\prime}$, provides a direct evidence (bearing in mind that function $A_{x}\left(y^{\prime}, z^{\prime}\right)$ is even in $\left.z^{\prime}\right)$ that integral (6) is zero for all values of $y$ satisfying the inequality $|y| \leq 1-|b|$. In the region $1<|y| \leq 1-|b|$ integral (6) leads to appearance of nonzero terms that for thin films are small due to the presence of the corrections of $(d / \lambda)^{n}(n>1)$ type. They have to be taken into account when we are interested in, for example, the distribution of the derivative $d \bar{A} / d y$ near the film edges (since disregard for these terms will cause a logarithmic divergence of the first derivative). For thick films, allowance for the near-edge regions of a superconductor in integral (6) cannot largely affect the $\bar{A}(y)$ distribution off the film edges because of smallness of $|b|$.

Thus, the $2 \mathrm{D}$ equation (3) is reduced to a $1 \mathrm{D}$ equation for the film-thickness-averaged vector potential $\bar{A}(y)$, which is valid in the region $|y| \leq 1-|b|$

$$
\bar{A}(y)=-H_{\infty} y W / 2+\frac{W d}{4 \pi \lambda^{2}} \int_{-1}^{1} \ln \left|y-y^{\prime}\right| \bar{A}\left(y^{\prime}\right) d y^{\prime}
$$

In the following sections the results of a numerical solution of Eqs. (7) and (3) are provided for thin and thick films.

\subsection{Thin films $(d<\lambda)$}

It turns out that in films satisfying the condition $d / \lambda \leq 1 / 4$ the difference between the solutions of Eqs (7) and (3) (averaged over thickness) is about $1 \%$ far from film edge and less than $4 \%$ in a narrow near-edge region. An appreciable error in the near-edge region (which is slightly growing towards the film edge with a larger numerical step) depends on the presence of the small corrections in (7) that were ignored.

Besides, the vector potential in this case is practically independent of $z$ (but not the derivative $\partial A_{x}(y, z) / \partial z$ ). Thus, at $d=\lambda$ the relation $A_{x}(y, 1) / A_{x}(y, 0) \approx 1.07$, i.e., the difference is about $7 \%$. With a lower $d / \lambda$ ratio the relation $A_{x}(y, 1) / A_{x}(y, 0)$ tends to unit.

We have derived an asymptotic expression for the vector potential distribution, satisfying Eq.(7) (and, hence, (3) averaged over $z$ ) with a sufficently high accuracy (not less than $3 \%$ at a film edge and far from edge region, and not less than $6 \%$ in the near-edge region, see Fig.2):

$$
\bar{A}(y)=-\frac{\lambda_{e f f} H_{\infty} y}{\sqrt{\alpha\left(1-y^{2}\right)+\beta}}
$$

where $\lambda_{\text {eff. }}=\lambda^{2} / d, \beta \simeq 2 \lambda_{\text {eff }} / \pi W+4\left(\lambda_{e f f} / W\right)^{2}$, and the dependence $\alpha\left(W / \lambda_{\text {eff }}\right)$ is shown in Fig.3 together with the approximation (9) 


$$
\alpha \approx 0.25-\frac{0.63}{\left(W / \lambda_{e f f}\right)^{0.5}}+\frac{1.2}{\left(W / \lambda_{e f f}\right)^{0.8}} .
$$

At $W<\lambda_{\text {eff }}$ the dependence $\bar{A}(y)$ becomes almost linear. Formula (8) with $\alpha=0.25, \beta=0$ was derived analytically in [1] by solving Eq.(7) (to be more exact, a simplified version of Eq.(7) in which the left-hand part is omitted, which corresponds to the condition $\left.W \lambda_{\text {eff }} \gg 1\right)$.

The resultant dependence $\bar{A}(y)$ allows to calculate the field concentration parameter $\gamma=\bar{H}_{z}^{\text {edge }} / H_{\infty}$ (where $\bar{H}_{z}^{\text {edge }}$ is the edge field averaged over superconductor thickness) for films of such type:

$$
\gamma=\frac{\bar{H}_{z}^{\text {edge }}}{H_{\infty}}=\frac{2 \lambda_{\text {eff }}}{W \sqrt{\beta}}\left(\sqrt{1+\frac{\alpha}{\beta}}\right)
$$

At $W \gg \lambda_{\text {eff }}$ Eq.(10) transforms into

$$
\gamma=\frac{\pi \sqrt{2 \pi}}{10} \sqrt{\frac{W}{\lambda_{e f f}}},
$$

where the coefficient preceding $\sqrt{W / \lambda_{\text {eff }}}$ is a quantity of order unit. The difference between Eq.(10) and the numerically obtained expression for $\gamma$ may reach 30\%: for wide films, $\left(W \gg \lambda_{e f f}\right)$, Eq.(10) yields an overestimated result, see insert in Fig.4. This takes place because, unlike the $\bar{A}(y)$ function itself, the first derivative (8) with respect to $y$ (magnetic field) adequately satisfies the numerical solution everywhere except for the narrow region near a film edge (see Fig.4). Due to the logarithmic divergence of the magnetic field at a film edge, which follows from the solution of Eq.(7), the approximation expression for $\bar{H}_{z}^{\text {edge }}$ was compared with the numerical solution of Eq.(3). Fig.4 also shows the interpolation function $1+0.66 \sqrt{W / \lambda_{e f f}}$ for the numerical analysis data (see solid line in the insert). Thus, the difference between (10) and the numerical result in the wide film limit $W / \lambda_{\text {eff }} \gg 1$ comes to about $17 \%$.

\subsection{Strip of finite thickness $(d \gg \lambda)$}

A comparative numerical analysis of the solutions to Eqs (3) integrated over a superconductor thickness and (7) was also carried out for the case $\lambda \ll d \ll W$. It was found out that the solutions coincide (to the accuracy of about 3\%) in the region $|y|<1-d / W$ and ( quite surprisingly) in points $|y|=1$. In the near-edge region we observed a difference in the solutions of Eqs.(3) and (7).

An approximation equation has also been derived for the dependence $\bar{A}(y)$. It turned out to be exactly the same as the dependence (8) with the selection $\alpha \approx 0.25, \beta \approx 0.64 \lambda^{2} / d W$. One can easily see that the obtained values for $\alpha$ and $\beta$ practically coincide, to a numerical error, with those obtained for thin films at $\lambda^{2} / W d \ll 1$. Fig.5 shows the dependence $\bar{A}(y)$ derived from the solution to equation (3), and also Eq.(8). It is seen from the latter that maximum deviation of the solution to Eq.(3) from (8) (and, hence, from the solution to (7)) occurs in the region $y>1-d / W$, but in point $y=1$, however, both solutions coincide again. Thus, expression (8) provides an adequate description of the $\bar{A}(y)$ distribution (the difference from the numerical solution of Eq.(3) does not exceed $3 \%$ ) in the region $|y| \leq 1-d / W$ and in points $|y|=1$. This confirms the above statement that Eq.(7) describes well the distribution of the $\bar{A}(y)$ dependence in a film depth. Besides, Eq.(7) is apparently valid immediately at the edge of a superconductor as well, which, in our opinion, is quite surprising fact.

Fig.6 shows the distribution of the current density over a film cross-section and the distribution of the absolute value of local magnetic field $\left(|H|=\sqrt{H_{z}^{2}+H_{y}^{2}}\right)$ both inside and outside a film with dimensions $W=100 \lambda, d=10 \lambda$. As seen from Fig.6a,c, the magnetic field reaches its maximum at the corners (side edges) of a superconductor. At the equator $(y=1, z=0)$ the magnetic field is less intensive, but not appreciably smaller than the field at the corners (for comparison, at the corners $|H| \approx 4.1 H_{\infty}$, while in the middle of a side face $|H| \approx 2.9 H_{\infty}$ for the given parameters of film). It is easily seen that towards the film interior (along the $y$-axis) the magnetic field is practically uniform through the entire sample thickness, except for the near-surface areas with the thickness of order $\lambda$ where magnetic lines abruptly change direction. A similar behaviour is demonstrated by a current density (see figs. 6 b,d). It is proved numerically that both the current density and the magnetic field fall off towards a sample centre by a law similar to the exponential one, not only from the side faces but also from the top and bottom ones. So, in thick films $\lambda \ll d \ll W$ screening currents flow only 
in the near-surface layers of thickness about $\lambda$. On the same scale there is a decrease of a local magnetic field in superconducting samples of such type.

The numerical solution of Eq.(3) also provides a possibility to determine the field at a film edge. Unfortunately, unlike with thin films, Eq.(8) differs largely from the numerical result for the near-edge region (this discrepancy may reach 30\%). Therefore, the use of (8) in calculations of a thickness-averaged $z$-component of the magnetic field at a film edge certainly leads to a considerable error.

In Fig.7 the obtained numerical dependences $\bar{H}_{z}^{\text {edge }} / H_{\infty}$ (the $z$-component of magnetic field, averaged over thickness) and $H_{z}(1,0) / H_{\infty}$ (the $z$-component of magnetic field on equator) on $\sqrt{W / d}$ are presented. It is clearly seen that with a good accuracy the dependences are linear even for the $W / d$ values which are close to unit. Note that the coefficient of proportionality between $\bar{H}_{z}^{\text {edge }} / H_{\infty} \quad \sqrt{W / d}$ is equal to $\sim 1.03$, which practically is the same as its estimate $(\sim 1)$ found in [9]. Generally speaking, the value of this coefficient depends on a film thickness (or, rather, the ratio $d / \lambda$ ). Thus, the insert in Fig.7 illustrates relationships $\bar{H}_{z}^{\text {edge }} / H_{\infty}, H_{z}(1,0) / H_{\infty}$ for various values of film thickness and shape parameter $W / d=5$. It is seen that only the quantity $H_{z}(1,0)$ is practically independent of the ratio $d / \lambda$. The strongest dependence on film thickness is exhibited by $H_{z}(1,1)$ (it grows with the increase of the ratio $d / \lambda$ ). This results in a slight increase of the average field $\bar{H}_{z}^{\text {edge }}$ with a growth of film thickness (given the same $W / d$ ratio). However, for very thick films, $d \gg \lambda, \bar{H}_{z}^{\text {edge }} / H_{\infty}$ is supposed to practically cease to be dependent on $d / \lambda$. Indeed, in the limit of interest the equator field is independent of $d / \lambda$, while the corner field $H_{z}(1,1)$ increases as $\sqrt{W / \lambda}$ (which was derived from the expression $(11 \mathrm{c}, \mathrm{d})$ given below $))$. The sharpest variation of the magnetic field intensity occurs at a distance of order $\lambda$ from the top/bottom surfaces. Correspondingly, the contribution of those regions in $\bar{H}_{z}^{\text {edge }} / H_{\infty}$ will be of order $\lambda \sqrt{W / \lambda} / d=\sqrt{W / d} \sqrt{\lambda / d}$ and will become negligible with the increase of the ratio $d / \lambda$.

Besides the approximation expression for $\bar{A}(y)$, we have found numerical estimates for the vector potential in points $(1,1)$ (on a corner); $(1,0)$ (on the equator), and also the distribution of the vector potential (current density) over the upper/lower surfaces:

$$
\begin{gathered}
A_{x}(1,0) \simeq-H \sqrt{\frac{W}{d}} \lambda, \\
A_{x}(1,1) \simeq A_{x}(W / 2,0)\left(1+\frac{1}{\sqrt{16 \pi}} \frac{d}{\lambda}\right)^{1 / 2}, \\
A_{x}(y, \pm 1)=-\frac{\lambda_{\text {eff }} H_{\infty} y}{\sqrt{\alpha\left(1-y^{2}\right)+\beta}},
\end{gathered}
$$

where

$$
\alpha \simeq \frac{0.25}{\left(1+(d / \lambda)^{2} / 2 \pi\right)}, \beta \simeq \frac{2 \lambda_{e f f}}{\pi W(1+(d / \lambda) / \sqrt{16 \pi})} .
$$

It is easily seen that at $d \gg \lambda$ the vector potential in points $( \pm 1, \pm 1)$ will largely exceed its value in points $( \pm 1,0)$. Using expression (11c) which is similar to (8) with renormalized parameters $\alpha$, $\beta$, we can find the points (lines) on the upper and lower surfaces, where the vector potential will coincide in absolute value with that on the equator. Simple calculations show that these lines are at a distance $\sim d / \pi$ from the side surfaces of the strip having $\lambda \ll d \ll W$.

These results allow to confirm the assumption (see subsection 2.1) on the possibility of expanding $A_{x}\left(y^{\prime}, z^{\prime}\right)$ in the limits $(y-b, y+b)$. Indeed, in the case of thin films the vector potential (or current density in a mixed state; see Sec. 4) varies on scales much larger than $\lambda$ and, hence, than $d$ (see (8)). For thick films, the vector potential (current density) far from edges is finite only in the surface layers of thickness of order $\lambda$. At the same time, the scale of variation for $A_{x}(y, z)$ along $y$ off sample edges is macroscopic (see expressions (11)). Therefore, expansion (6) is also possible off the edges. Near the edges, however, $A_{x}(y, z) \neq 0$ over entire thickness, and the scale of $A(y, z)$ variation (see fig.6b,d) in this region is $\lambda$ (in the $y$ direction). Hence, expansion (6) is not valid in the limits $y-b \leq y^{\prime} \leq y+b$ near the edges $(|y| \rightarrow 1)$ of thick superconducting film. 


\section{The conditions for vortex entry in superconducting films}

Using expressions $(8,11)$ it is possible to estimate the edge barrier suppression field ${ }_{s}$ or the first-vortex entry field into superconducting films.

\subsection{Thin film}

The vortices may enter into a thin film in the Meissner state provided the condition $|\bar{A}( \pm 1)|=A_{\text {crit }}$ is met; here $A_{\text {crit }} \approx \Phi_{0} / 2 \pi \xi$ [11, 12] ( $\Phi_{0}$ is the quantum of a magnetic flux, $\xi$ is the coherence length). The resultant expression for $H_{s}$ is

$$
H_{s} \approx \frac{\Phi_{0}}{2 \pi \xi \lambda_{e f f}} \sqrt{\beta}
$$

with $\beta$ being the same as in the expression (8). Dependence (12) in the limit $W \gg \lambda_{\text {eff }}$ and $W \ll \lambda_{\text {eff }}$ coincides to a factor of order one with the expression for the Meissner state breakdown field obtained in the limiting cases of wide and narrow thin films in [1]. From $(10,12)$ we can easily find the value of the magnetic field (or, rather, the thickness-averaged z-component) at a film edge $\bar{H}_{z}^{\text {edge }}$, when vortices start penetrating in it. For example, for wide films $\bar{H}_{z}^{\text {edge }}$ is

$$
\bar{H}_{z}^{e d g e} \approx \frac{\Phi_{0}}{10 \xi \lambda_{e f f}}
$$

To an accuracy of the factor of order one the above expression coincides with the field in the core of a Pearl vortex which is equal to $\Phi_{0} / 4 \pi \xi \lambda_{\text {eff }}[13]$.

\section{$3.2 \quad$ Thick film}

The main difference between thick and thin films is that the vector potential for the former largely depends on $z$. However, we should apparently anticipate that the conditions of vortex entry here will be qualitatively similar to those for thin films. Indeed, as shown in 11, 12, after the vector potential has reached its critical value at a superconductor edge in the Meissner state (in the mixed state it is the gauge-invariant potential $\boldsymbol{\Pi}=\Phi_{0} \nabla \varphi / 2 \pi-\mathbf{A}$ that should reach a critical value), the order parameter $\Psi=|\Psi| e^{i \varphi}$ is strongly suppressed and vortex formation begins. The above papers dealt with bulk superconductors and thin-film samples, which, due to the symmetry of the problem or problem geometry, could be assumed homogeneous along the $z$-axis.

It should be expected that in thick films the order parameter will be suppressed in the regions where the vector potential $A_{x}(y, z)$ reaches its critical value.

First, the condition $\left|A_{x}(y, z)\right|=A_{\text {crit }}$ will be satisfied at the side edges $( \pm 1, \pm 1)$ of a superconductor (as the magnetic field is increasing from zero). It means that the order parameter will be suppressed in these points first. With a further increase of the magnetic field this situation may develop by two scenarios:

1. Suppression of the order parameter results in tilted vortices that start to form at the corners of a superconductor cross-section. When the vector potential at the equator reaches the critical value, two tilted vortices fragments (from top and from bottom) will join each other to form one rectilinear vortex. In the absence of pinning the latter is able to penetrate into the sample centre driven by the Lorentz force. Similar vortex entry scheme was discussed in [9].

2. In the course of further magnetic field increase, the order parameter becomes suppressed in the region of side edges. This causes areas with a suppressed order parameter to appear near the side edges, which would allow to regard a film cross-section as a rectangular with rounded-off edges. It should be emphasized that the geometrical sizes of sample remain unchanged in this situation, only its physical properties vary in the regions near side edges. This scenario allows to explain the physical mechanism behind the formation of the "geometrical rounds-off" near the corners of a rectangular cross-section sample, which were considered in [3]. However, unlike [3, 9], our approach is based on the assumption that vortices will start entering deep inside a superconductor when the condition $\left|A_{x}(y, z)\right|=A_{\text {crit }}$ is fulfilled at the equator. By that moment the effective "round-off" radius will reach a value of order $d / 2$.

Which of these two scenarios is practically feasible can be found out only through experiment. Theoretically this question can be answered by numerical solution of a problem on a vortex entry in a 3D sample within the nonstationary theory of Ginzburg-Landau. 
The feature common for the above two schemes is, actually, the assumption that vortices enter deep a thick film when the condition $\left|A_{x}(1,0)\right|=A_{\text {crit }}$ is met. This allows to estimate the field of vortex entry inside a sample. Using Eq.(11a) (regardless of possible variation due to the penetration of tilted vortices or the existence of areas with a suppressed order parameter), we now derive the expression for field $H_{s}$, which is equivalent (12) (with $\beta=2 \lambda_{\text {eff }} / \pi W$ ). This similarity is due to the fact that the $A_{x}( \pm 1,0)$ value is defined practically by the same expression for both thin and thick films, provided parameter $\lambda^{2} / d W$ is the same.

By analogy with thin films, one can find a value of the field at a film edge when the vortices start to penetrate

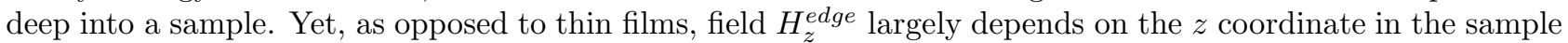
region. Estimations of the equator field yields

$$
H_{z}^{e q}=H_{z}(1,0) \approx \frac{\Phi_{0}}{2 \pi \xi \lambda}
$$

which is practically equivalent to the $\bar{H}_{z}^{\text {edge }}$ (see fig.7). Note also that $H_{z}^{e q}\left(H_{s}\right)$ to the factor of order unit is equal to the thermodynamical field $H_{c}$, or the surface barrier suppression field for bulk superconductors in the absence of edge defects.

\subsection{The influence of surface defects and anisotropy}

The resultant values for field $H_{s}$ are characteristics of isotropic superconductors with ideal surface. As was established in [12, 14], surface defects can considerably decrease the value of $H_{s}$. For example, in [14] for the case $\kappa \gg 1$ ( $\kappa$ is the Ginzburg-Landau parameter) maximum suppression of the entry field $g=H_{s} / H_{e n}$ (where $H_{e n}$ is the field of vortices entry in a superconductor with surface defects) was estimated as $g \approx \sqrt{\kappa / \pi}$. Thus, for $\kappa=100$ we will have $g \simeq 5.6$.

A strong influence on the value of $H_{s}$ may be produced also by anisotropy or, rather, layered structure of superconductors. If the layers are not Josephson-coupled (or are weakly coupled), a superconductor should be regarded as a stack of superconducting layers. This geometry can be simulated, if we multiply the integrand in equation (3) (and, hence, in integral (6)) by the step periodic function $z$ which is equal to one in the superconducting layer and is zero in the interlayer space.

Let a period in such a structure be much smaller than $\lambda$ (which is practically always fulfilled for HTSC), a layer thickness be $l$ and an interlayer separation be $m$. Then we can assume the distribution of $A_{x}(z)$ to be a smooth function $z$, similar to the dependence $A_{x}(z)$ for a homogeneous superconductor. In this case, the integral in Eq.(3) for a layered superconductor will be $(l+m) / l$ times smaller than that for an isotropic superconductor. In other words, we can replace this integral for a layered superconductor by the integral for an isotropic case by introducing an effective penetration depth $\lambda^{\prime}=\lambda \sqrt{(l+m) / l}$. In this way we immediately obtain the distributions of $\bar{A}(y), A_{x}(y, 1)$, and also the values for $A_{x}(y, z)$ at the side edges and the equator with allowance for the layered structure of superconductor.

One particular effect of the anisotropy is that the value of $A_{x}(1,0)$ will be $\sqrt{(l+m) / l}$ times larger than that for an isotropic superconductor, all other conditions being equal. This, in its turn, will lead to a $\sqrt{(l+m) / l}$ times smaller field of vortex entry in a superconductor. For example, at $l=3 \dot{A}$ and $m=12 \dot{A}$, typical for $B i B a C u O$, one finds $\sqrt{(l+m) / l} \approx 2.2$.

Thus, the two above-mentioned factors, i.e., surface defects and layered structure of superconductors may cause a considerable (10-fold and more) decrease of the vortex entry field in layered superconductors with surface defects, as compared to the vortex entry field in isotropic perfect-surface superconducting films.

Another conclusion following from the fact of a layered structure in such systems is that the scale of a local magnetic field penetration, for example, for thick films, will be $\lambda^{\prime}=\lambda \sqrt{(l+m) / l}$, and for thin films the parameter $\lambda_{\text {eff }}$ has to be changed for $\lambda_{\text {eff }}^{\prime}=\lambda^{\prime 2} / d$. At the same time, the thickness-averaged current density, unlike the thickness-averaged vector potential, will practically remain unchanged across the entire film width, except for the regions lying close to sample edges. This can be accounted for by the fact that the expression for current density $j(y)=-c A(y) / 4 \pi \lambda^{2}$ will include $\lambda$ only at a superconductor edge (see (8)). Likewise, all other quantities that obviously do not include $\lambda$ (for example, a degree of the magnetic field concentration at the equator of thick film) will remain the same. 


\section{The structure of a mixed state}

Let us now discuss the parameters of a mixed state arising in a superconducting film in fields larger than $H_{s}$. Here we neglect the presence of bulk pinning, which is justified for soft superconductors. This problem was studied earlier in [3, 5, 6, 7, 9, 15]. In [7, 15] the authors considered a case of narrow thin films $W d / \lambda^{2} \ll 1$, [5] deals with wide thin films $W d / \lambda^{2} \gg 1$, and [3, 9] is a study on thick films that formally obey the condition $W d / \lambda^{2} \gg 1$. We will analyse a general case to show that it embraces either of the above two situations. Besides, the resultant distribution of current density will be finite across the entire film width, as opposed to the results of the above cited works.

Consider a superconducting film in a mixed state, placed in a perpendicular magnetic field. The current density and the vector potential in the London limit are related as $\mathbf{j}=-\left(\mathbf{A}-\Phi_{0} \nabla \varphi / 2 \pi\right) c / 4 \pi \lambda^{2}$, where $\varphi$ is the order parameter phase. The Maxwell equation will have the form (1), in which $\Delta$ is now a 3D Laplacian operator. Using the Green function for $\Delta$, we can write this equation in an integral form:

$$
\mathbf{A}(\mathbf{r})=\mathbf{A}^{0}(\mathbf{r})+\frac{1}{c} \iiint \frac{\mathbf{j}\left(\mathbf{r}^{\prime}\right)}{\left|\mathbf{r}-\mathbf{r}^{\prime}\right|} d x^{\prime} d y^{\prime} d z^{\prime}
$$

We now subtract function $\nabla \varphi(\mathbf{r})$ from the left- and right-hand parts of Eq.(13) and take curl from these parts (using the property $\nabla \times \nabla \varphi(\mathbf{r})=2 \pi \delta\left(\mathbf{r}-\mathbf{r}^{\prime}\right)$, where $\mathbf{r}^{\prime}=\left(x^{\prime}, y^{\prime}\right)$ are the vortex coordinates). Next, we do the averaging over coordinates $x, y$ on scales much larger than an intervortex distance. After these operations, the distribution of a current density becomes uniform along the $x$-axis and we can perform integration over $x^{\prime}$ in (13). Then we average the obtained equation over a film thickness and use the results of the integral (6) calculations. This will yields an equation for the sheet current density $i(y)=\int_{-d / 2}^{d / 2} j_{x}(y, z) d z$,

$$
\frac{8 \pi \lambda_{e f f}}{c W} \frac{d i(y)}{d y}+\frac{2}{c} \int_{-1}^{1} \frac{i\left(y^{\prime}\right)}{y-y^{\prime}} d y^{\prime}=-H_{\infty}+n(y) \Phi_{0}
$$

where $n(y)$ is the density of vortices, the distance being measured in units of $W / 2$. For the first time this equation was derived in [4] for thin films $d \ll \lambda$. Just like Eq.(7), (14) is valid at $|y| \leq 1-|b|$ for thick films, and across the width for thin films (excluding an extremely narrow near-edge region of size $d \ll \lambda$ ). Besides, it should be expected by analogy with the Meissner state that Eq.(14) for thick films will also be valid directly at a sample edge.

We analysed Eq.(14) numerically for different values of parameter $W / \lambda_{\text {eff }}$, using the condition that current density is zero in the region where vortices exist, and takes finite value in vortex-free regions [3, 5, 6, 95. Besides, we set a boundary condition $j( \pm 1)= \pm j_{s}$ on a current density (in increasing magnetic field), which allows for an edge barrier. The value of the current density $j_{s}$ of order of the Ginzburg-Landau depairing current density for ideal-surface superconductors 11, 12. In result, we have obtained the approximation expression for $i(y)$

$$
i(y)= \begin{cases}0 & 0<|y|<a, \\ \frac{c H_{\infty}(z+1)}{4 \pi \sqrt{\alpha\left(1-z^{2}\right)+\beta \frac{(|y|+a)^{2}}{(1+a)^{2}}}} \operatorname{sign}(y) & a<|y|<1,\end{cases}
$$

where

$$
\begin{gathered}
z=\frac{2\left(y^{2}-a^{2}\right)}{\left(1-a^{2}\right)}-1, \\
\beta \simeq \frac{8}{\pi} \frac{1}{1-a^{2}} \frac{\lambda_{\text {eff }}}{W}+\frac{16}{(1-a)^{2}}\left(\frac{\lambda_{\text {eff }}}{W}\right)^{2},
\end{gathered}
$$

$a\left(H_{\infty}\right)$ is the half-width of the vortex-filled region and parameter $\alpha$ is defined by expression (9) in which $\mathrm{W}$ has been replaced by $W(1-a)$.

Expressions (15), being not derived, represents a rather useful interpolation for the distribution of the sheet current density for a film in a mixed state. We would like to emphasize again that in the thin-film case $W / \lambda_{\text {eff }}$ can be both smaller (narrow films) and larger (wide films) than unit, whereas for thick films this ratio is always much larger than 1. 
Fig. 8 shows the dependence $i(y)$ for different values of a magnetic field. The difference of approximation (15) from the numerical result does not exceed $4 \%$ in the vortex-free zone $(a<y<1)$. Note, that in the near-edge region and close to the boundary of the vortex-filled region deviation may come to about $9 \%$.

The dependence $a\left(H_{\infty}\right)$ (in increasing magnetic field) is to be found from the following expression:

$$
\frac{8}{\pi} \frac{1}{1-a^{2}} \frac{\lambda_{e f f}}{W}+\frac{16}{(1-a)^{2}}\left(\frac{\lambda_{e f f}}{W}\right)^{2}=\left(\frac{H_{\infty}}{H_{s}}\right)^{2}\left(\frac{8}{\pi} \frac{\lambda_{e f f}}{W}+16\left(\frac{\lambda_{e f f}}{W}\right)^{2}\right) .
$$

For $W / \lambda_{\text {eff }} \gg 1$ we have

$$
a\left(H_{\infty}\right)=\sqrt{1-\left(H_{s} / H_{\infty}\right)^{2}}, \quad H_{\infty} \simeq H_{s},
$$

or

$$
a\left(H_{\infty}\right)=1-\sqrt{\frac{2 \lambda_{e f f}}{\pi W}} \frac{H_{s}}{H_{\infty}}, \quad H_{\infty} \gg H_{s},
$$

while for $W / \lambda_{e f f} \ll 1$ we have

$$
a\left(H_{\infty}\right)=1-H_{s} / H_{\infty}
$$

for all values of $H_{\infty}$.

Using dependence (15), we can find distribution of the $z$-averaged magnetic field across a film width:

$$
\bar{H}_{z}(y)=H_{\infty}+\frac{2}{c} \int_{-1}^{1} \frac{i\left(y^{\prime}\right)}{y-y^{\prime}} d y^{\prime} .
$$

Fig. 9 shows the dependences $\bar{H}_{z}(y)$ for a film with $W / \lambda_{\text {eff }}=200$ and $a=0.6\left(H_{\infty} \simeq 1.3 H_{s}\right)$, obtained numerically and by means of expression $(15,16)$. It is seen that these dependences practically coincide across the entire width of a film, except for the near-edge regions and the boundary of the vortex-filled zone. The difference in the magnetic field value at $y=0.6$ should be attributed to the inaccuracy of approximation (more precisely, its first derivative at the boundary of the vortex-filled area). Dependence $\bar{H}_{z}(y)$ shown in the insert to Fig. 9 was obtained theoretically in [3, 5, 9]. It is seen that, as opposed to this analytical dependence, a non-zero magnetic field does exist outside the vortex-filled region also, and it is quite strong $\left(>0.1 H_{\infty}\right)$ for a film with the given parameters. Another distinguishing feature is the occurrence of a jump from zero to some finite value for the dependence $n(y)=\bar{H}_{z}(y) / \Phi_{0}$ at $y=a$. The reason of the vortex density discontinuity is explained by a non-zero magnetic field in the region $(a, 1)$ and the condition of a magnetic field continuity at $y=a$.

Using Eq.(15), we can estimate the dependence of $\bar{H}_{z}^{\text {edge }} / H_{\infty}$ on the parameters of a film and an increasing external magnetic field (for thin films; see subsection 2.2)

$$
\frac{\bar{H}_{z}^{\text {edge }}}{H_{\infty}}=\frac{2 \lambda_{\text {eff }}}{W} \frac{1}{\sqrt{\beta}}\left(1+\frac{1}{\beta}\left(2 \alpha-\frac{\beta(1-a)}{2 \sqrt{1+a^{2} / 2}}\right)\right) \frac{4}{1-a^{2}} .
$$

It is nicely seen that in the limit $a \rightarrow 1\left(H_{\infty} \rightarrow \infty\right)$ the ratio $\bar{H}_{z}^{\text {edge }} / H_{\infty}$ tends to 1 .

Knowing the dependence $i(y)$, we can find the magnetization curves of superconducting films for different values of $W / \lambda_{\text {eff }}$. Fig.10 illustrates the obtained results. One can see that with a increasing parameter $W / \lambda_{\text {eff }}$ the magnetization curves become similar to the dependence $M(H)$ derived theoretically in $\| 5$ for wide thin films. As $W / \lambda_{\text {eff }}$ decreases, the magnetization curves tend to the dependence which is valid for thin narrow films [7, 15]. Thus, even at $W / \lambda_{e f f}=1$ the dependence $M(H)$ for a narrow film and the $M(H)$ obtained numerically by the use of Eq.(15) practically coincide. So, expression (15) allows to obtain magnetization curves for such superconductors at arbitrary ratio $W / \lambda_{e f f}$. Note that the magnetization curves in this case, i.e., at any value of parameter $W / \lambda_{\text {eff }}$ lie between two curves - 1 and 3 as shown in Fig. 10 (in dimensionless units). 


\section{Conclusion}

It is shown in the present paper that the Maxwell-London equation used so far only for thin films is also valid for samples of finite thickness. This equation is shown to define the distribution of a thickness-averaged vector potential and/or current density (in the mixed state case) across a sample width. For thin films the equation holds practically everywhere in a film, whereas in the thick film case its applicability is restricted only within a narrow bands near the edges $W / 2-d / 2 \leq|Y| \leq W / 2$.

An approximation expression is found, describing distribution of vector potential $\bar{A}$ (or current density $\bar{j}(y)$ ) across the width of a film in the Meissner state, which applies to both thin and thick films. For thick films analytical expressions have been derived, defining the value of the vector potential (local current density) at the equator $(Y=1, Z=0)$, side edges $(Y=1, Z=1)$, and also on the top and bottom surfaces $(Y, Z= \pm d / 2)$ of a sample. Besides, analytical approximation expressions have been found for the magnetic field at the equator and for the thickness-averaged edge magnetic field.

The vector potential distribution data were used to evaluate the field of the the first vortex entry (barrier suupresion field) for superconductors of such geometry. It's described by an universal expression (12) valid for both thin and thick films. It is shown that besides surface defects the layered structure of superconductor may result in a significant (up to 100\%) suppression of the vortex entry field. Thus, mutual influence both surface defects and layered structure may lead to suppression of $H_{s}$ by factor ten (and even greater).

The study of a mixed state yields an interpolation expression for distribution of a sheet current density $i(y)$ in superconducting films without bulk pinning. This result allows to for the first time estimate the dependence on the magnetic field concentration $\gamma=\bar{H}_{z}^{\text {edge }} / H_{\infty}$ on the parameters of superconductor and external magnetic field $H_{\infty}$. Besides, these data were used to calculate the magnetization curves for film superconductors at any values of parameter $W d / \lambda^{2}$.

\section{Acknowledgements}

Authors are obliged to Prof. J.R.Clem, Dr. G.M. Maksimova for helpful discussions of the results obtained. This work is supported by the Science Ministry of Russia (Project No. 98-012), and in part Basic Foundation for Fundamental Research (Project No. 97-02-17437). Partial support of the International Center for Advanced Studies (INCAS) through Grant No. 99-2-03 is gratefully acknowledged.

\section{References}

[1] K.K.Likharev, Izv. vuz. Radiofizika 14 (1971) 909 [Radiophys. Quantum Electron. 14 (1971) 714]; 14 (1971) 919 [14 (1971) 722].

[2] E.H.Brandt, M.Indenbom, Phys. Rev. B 48 (1993) 12893; E.H. Brandt, Phys. Rev. Lett., 71 (1993) 2821; E.H. Brandt, Phys. Rev. B, 59 (1999) 3369 and references in.

[3] M.Benkraouda, J.R.Clem, Phys. Rev. B 53 (1996) 5716; Phys. Rev. B 58 (1998) 15103;

[4] A.I.Larkin, Yu.N.Ovchinikov, Zh. Eksp. Teor. Fiz. 61 (1971) 1221 [Sov.Phys. JETP 34 (1972) 651].

[5] I.L.Maksimov, Techn.Phys.Lett. 22, n 20 (1996) 56.

[6] I.L.Maksimov, A.Elistratov, Appl.Phys.Lett. 72 (1998) 1650; I.L. Maksimov, A. Elistratov, JETP Lett. 61, 208 (1995).

[7] G.M.Maksimova, Phys. of Sol. State 40 (1998) 1773.

[8] L.G.Aslamazov, S.V.Lempickii, Zh. Eksp. Teor. Fiz., 84 (1983) 2216.

[9] E. Zeldov, A.I. Larkin, V.B. Geshkenbein, M. Konczykowski, D. Majer, B. Khaykovich, V.M. Vinokur, and H. Shtrikman, Phys. Rev. Lett. 73 (1994) 1428. 
[10] M. Wurlitzer, M. Lorenz, K. Zimmer, P. Esqunazi, Phys. Rev. B, 55 (1997) 11816; M. Konczykowski, L.I. Burlachkov, Y. Yeshurun, F. Holtzberg, Phys. Rev. B 43 (1991) 13707; L.I. Burlachkov, Y. Yeshurun, M. Konczykowski, F. Holtzberg, Phys. Rev. B 45 (1992) 8193; H. Castro, B. Dutoit, A. Jacquier, M. Baharami, and L. Riuderer, Phys. Rev. B 59 (1999) 596; V. Jeudy and D. Limagne, Phys. Rev. B, 60 (1999) 9720; M.E. Gaevski, A.V. Bobyl, D.V. Shantsev, Y.M. Galperin, T.H. Johansen, M. Baziljevich, H. Bratsberg, S.F. Karmanenko, Phys. Rev. B 59 (1999) 9655; F.Mrowka, M. Wurlitzer, P. Esquinazi, E. Zeldov, T. Tamegai, S. Ooi, K. Rogacki, B. Dabrowski, Phys. Rev. B 60 (1999) 4370;

[11] D.Yu.Vodolazov, I.L.Maksimov, E.H.Brandt, Europhys. Lett. 48 (1999) 313.

[12] D.Yu.Vodolazov, I.L.Maksimov, E.H.Brandt, unpublished.

[13] J.Pearl, Appl.Phys.Lett. 5 (1964) 65.

[14] A.Yu.Aladyshkin, A.S.Mel'nikov, I.A.Shereshevsky and I.D.Tokman, cond-mat/9911430.

[15] G.M. Maksimova, D.Yu. Vodolazov, M.V. Balakina, I.L. Maksimov, Sol. St. Comm. 111, 367 (1999). 


\section{Figure captions}

fig.1

Geometry of the problem.

fig.2

Distribution of averaged vector potential, for different ratios $W / \lambda_{\text {eff }}$ : curves 1-5 for $W / \lambda_{\text {eff }}=1,5,10,50,200$, respectively. Dotted lines are numerical results, solid lines are approximation (8).

fig.3

Dependence of the parameter $\alpha$ on $W / \lambda_{e f f}$. Circles are numerical results, solid curve 1 is the approximation (9).

fig.4

Distribution of $\bar{H}_{z}$ inside the film for $W / \lambda_{e f f}=200$. Dots are numerical result, solid line is expression obtained from approximation (8). Insert shows dependence $\bar{H}_{z}^{\text {edge }}\left(W / \lambda_{\text {eff }}\right)$ : circles are numerical result, dotted line is the expression (10), solid line is the fitting function $1+0.66 \sqrt{W / \lambda_{e f f}}$.

fig.5

Distribution of averaged vector potential at thick film $(d=10 \lambda)$ for various widths: $W=50 \lambda(1), W=100 \lambda$ (2), $W=200 \lambda(3)$. Dots are numerical result, solid lines are expression (8) with $\alpha=0.25, \beta=2 \lambda^{2} / \pi d W$.

fig.6

Contour lines of the intensity of magnetic field (a,c) and current density (b,d) of thick $(W=100 \lambda, d=10 \lambda)$ film in applied perpendicular magnetic field. The step for magnetic field is $0.41 H_{\infty}$, for current density is $0.1 j(1,1)$. Maximum values of magnetic field $\left(H=4.1 H_{\infty}\right)$ and current density $(j=1)$ are reached at the corners of the film.

fig.7

Dependences $\bar{H}_{z}^{\text {edge }}$ (circles) and $H_{z}(1,0)$ (dots) on the parameter $\sqrt{W / d}$. Solid line 1 is the fitting function $1 / 3+1.03 \sqrt{W / d}$, dotted line 2 is the fitting function $1 / 3+0.92 \sqrt{W / d}$. Insert shows the dependences of $\bar{H}_{z}^{\text {edge }}$ (circles), $H_{z}(1,0)$ (dots) and $H_{z}(1,1)$ (stars) are shown on the film thickness for $W / d=5$.

fig. 8

Distribution of the sheet current $i(y)$ for film with parameter $W / \lambda_{\text {eff }}=200$ and for different values of $a$ : 0.0 (1), 0.4 (2), 0.8 (3). Dotted lines are numerical results, solid lines are expression (15).

fig.9

Distribution of the averaged $z$-component of magnetic field for $W / \lambda_{\text {eff }}=200$ and $a=0.6$. Solid line is obtained with help of expression $(15,16)$, dotted line is numerical result. Insert shows detailed distribution of the field; dashed line is the function $H \sqrt{a^{2}-y^{2}} / \sqrt{1-y^{2}}$ from [5, 9.

fig.10

Magnetization curves of superconducting films for different ratio $W / \lambda_{\text {eff }}$ : curve 1 for $W / \lambda_{\text {eff }}=\infty$, curve 2 for $W / \lambda_{\text {eff }}=200$, curve 3 for $W / \lambda_{\text {eff }}=1$. Curve 3 practically coincides with the magnetization curve for narrow $W \ll \lambda_{\text {eff }}$ films [15]. 


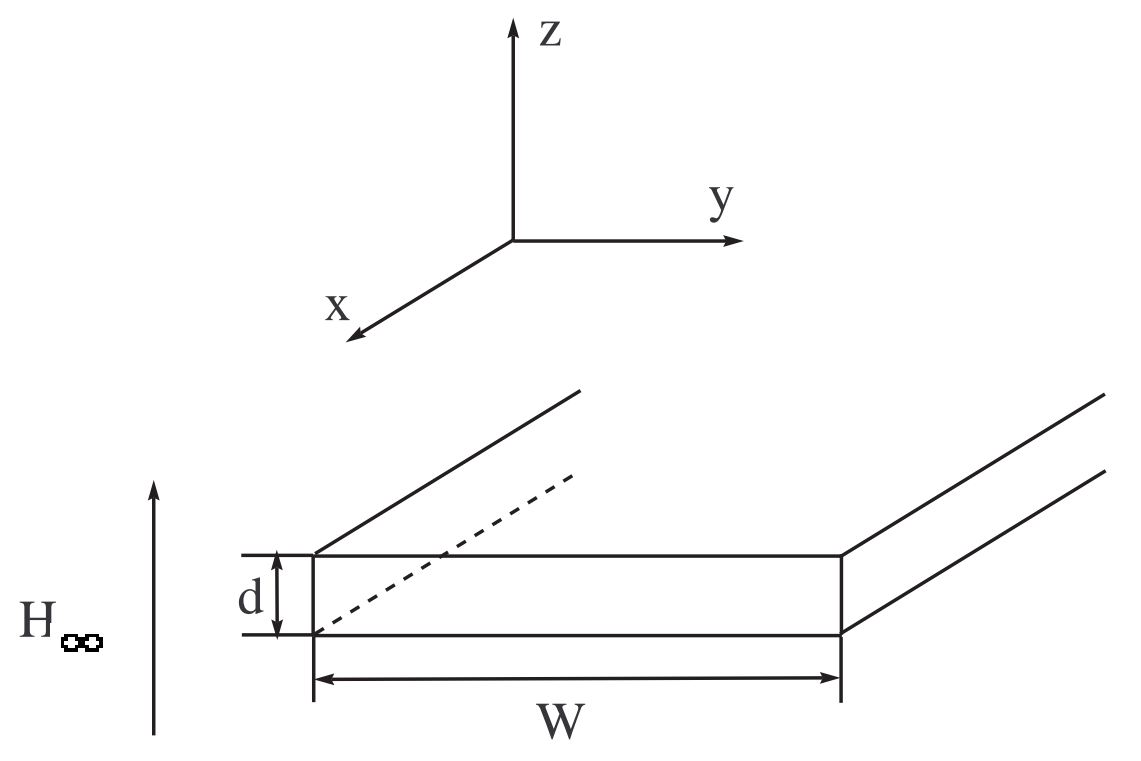

fig. 1 D.Yu.Vodolazov and I.L.Maksimov 


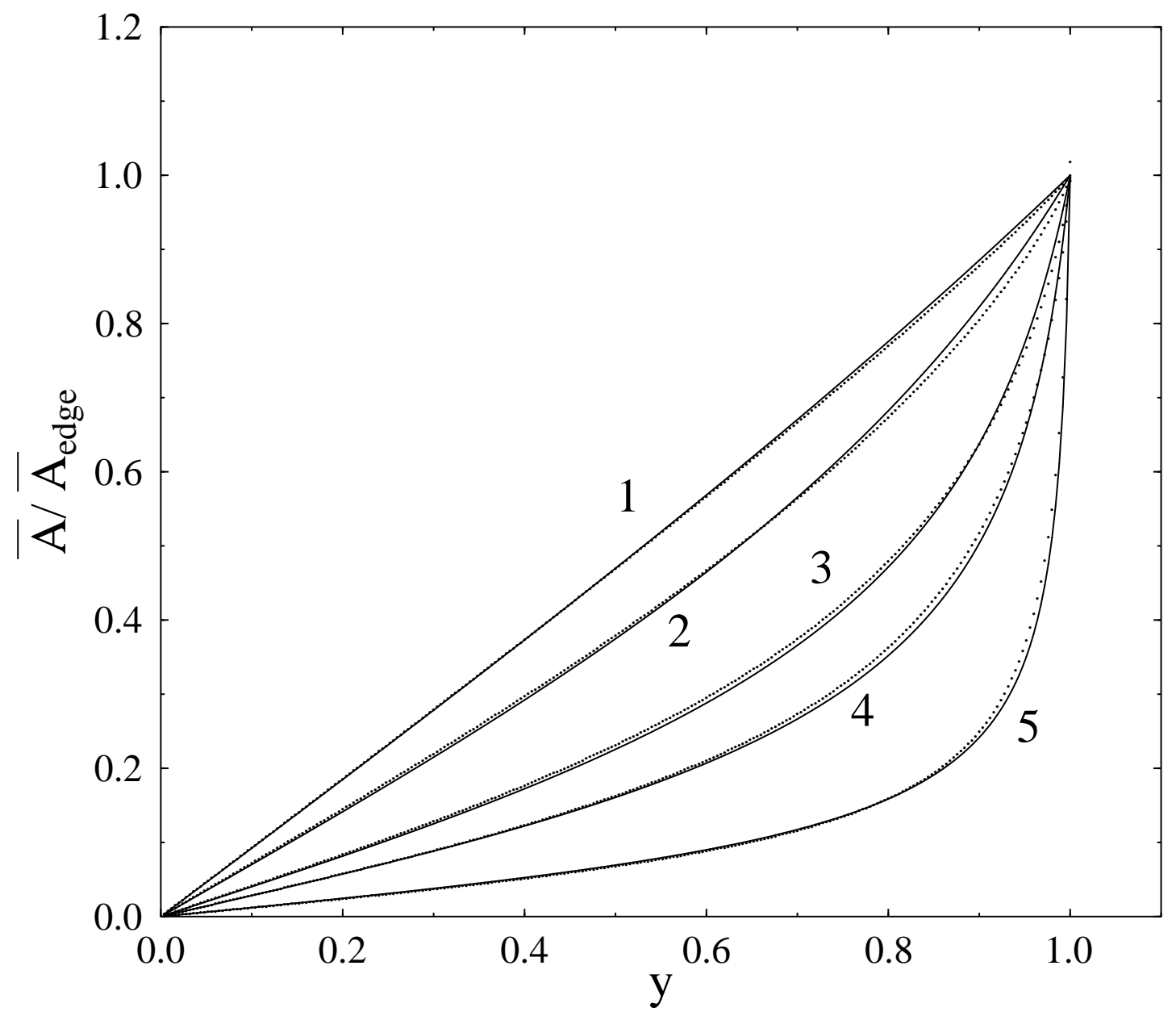

fig. 2 D.Yu.Vodolazov and I.L.Maksimov 


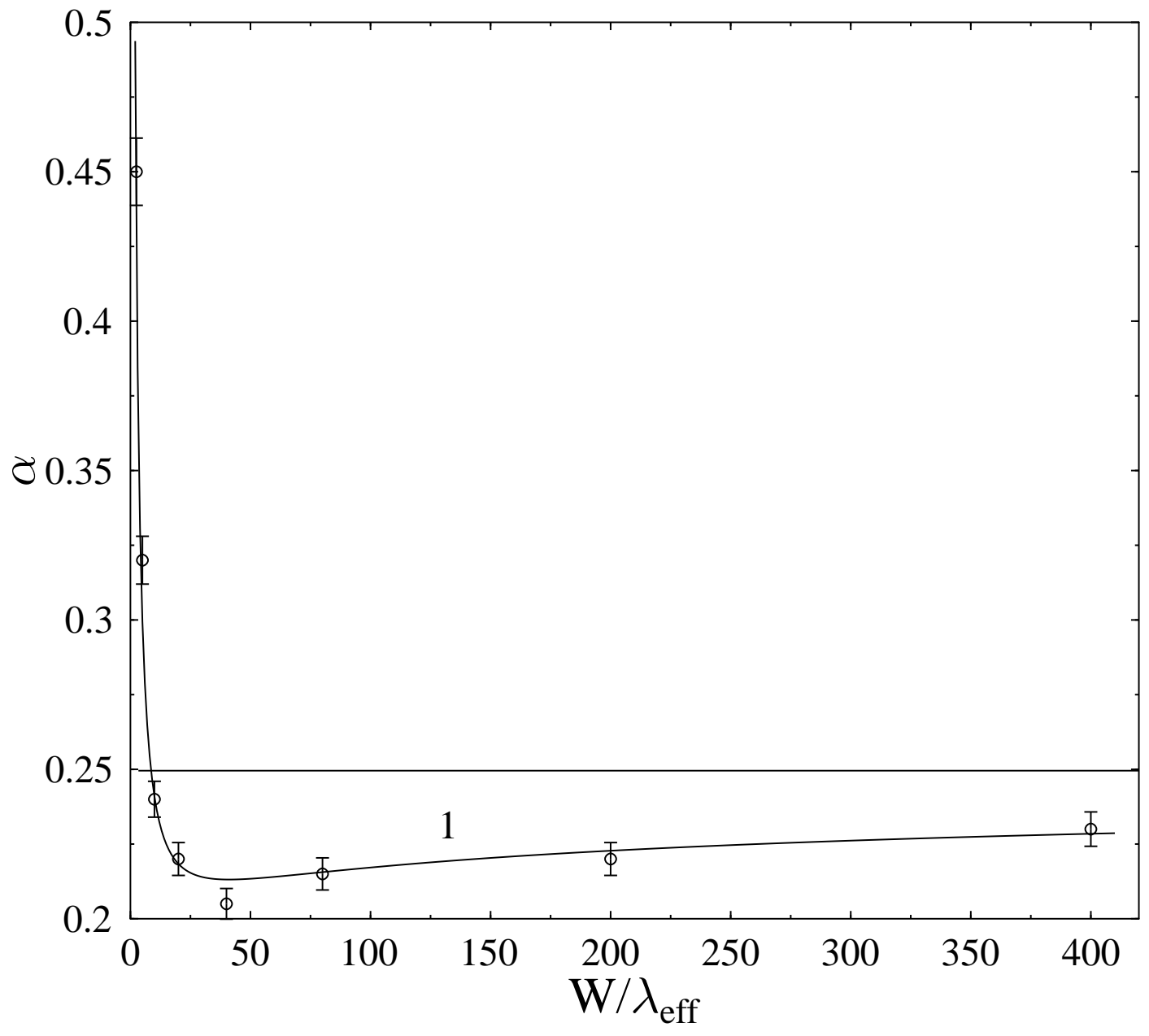

fig.3 D.Yu. Vodolazov and I.L.Maksimov 


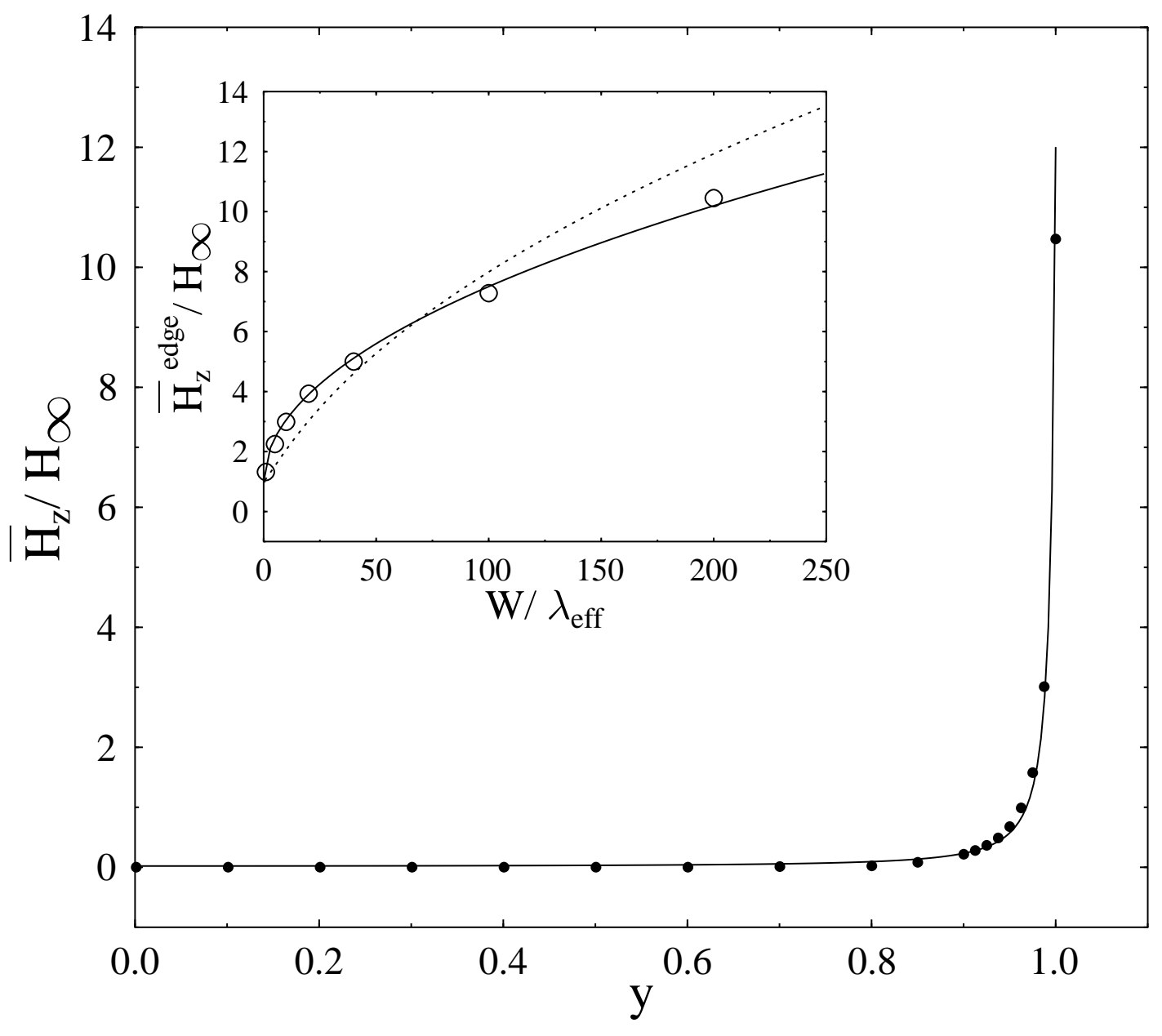

fig.4 D.Yu.Vodolazov and I.L.Maksimov 


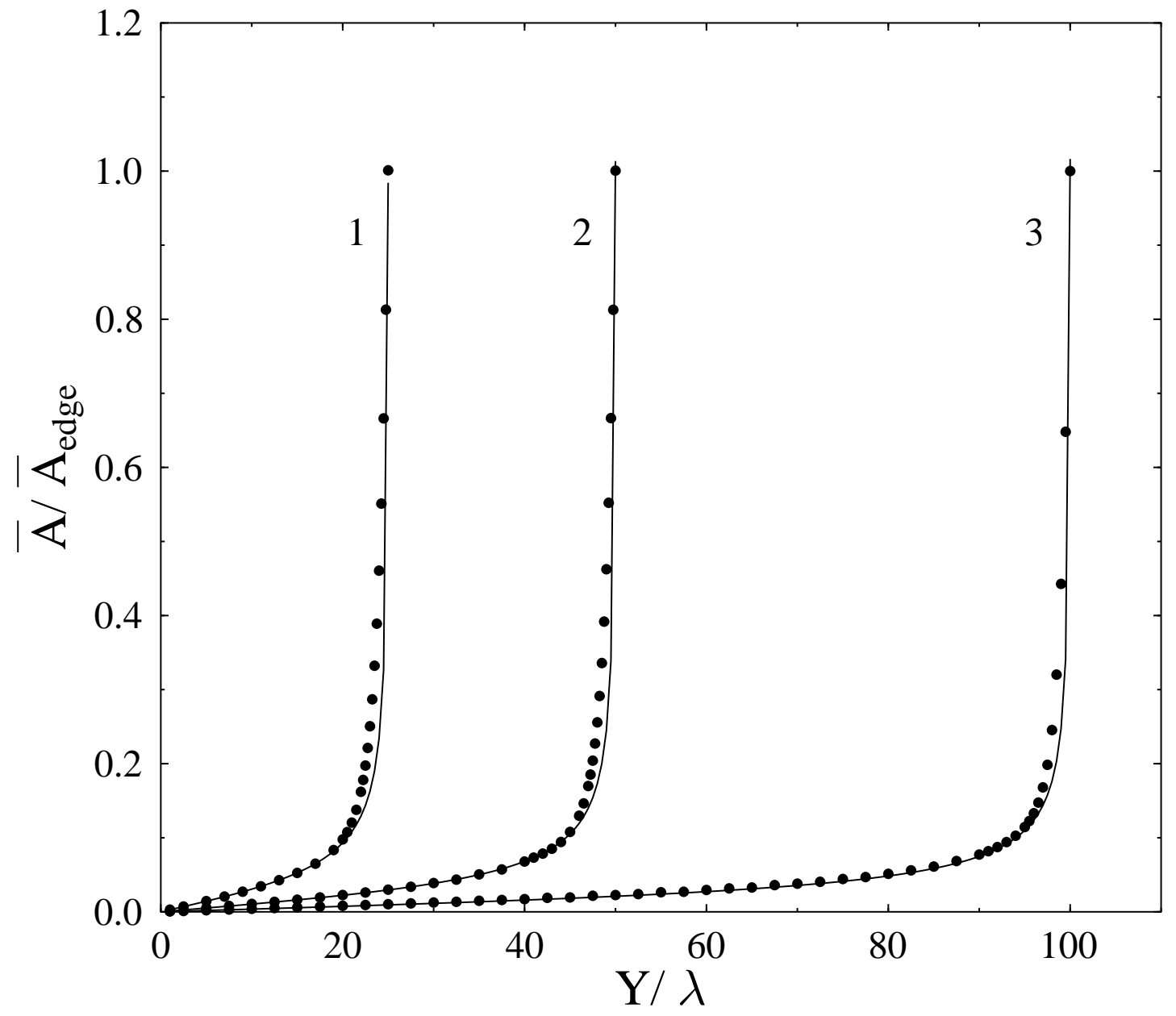

fig. 5 D.Yu.Vodolazov and I.L.Maksimov 

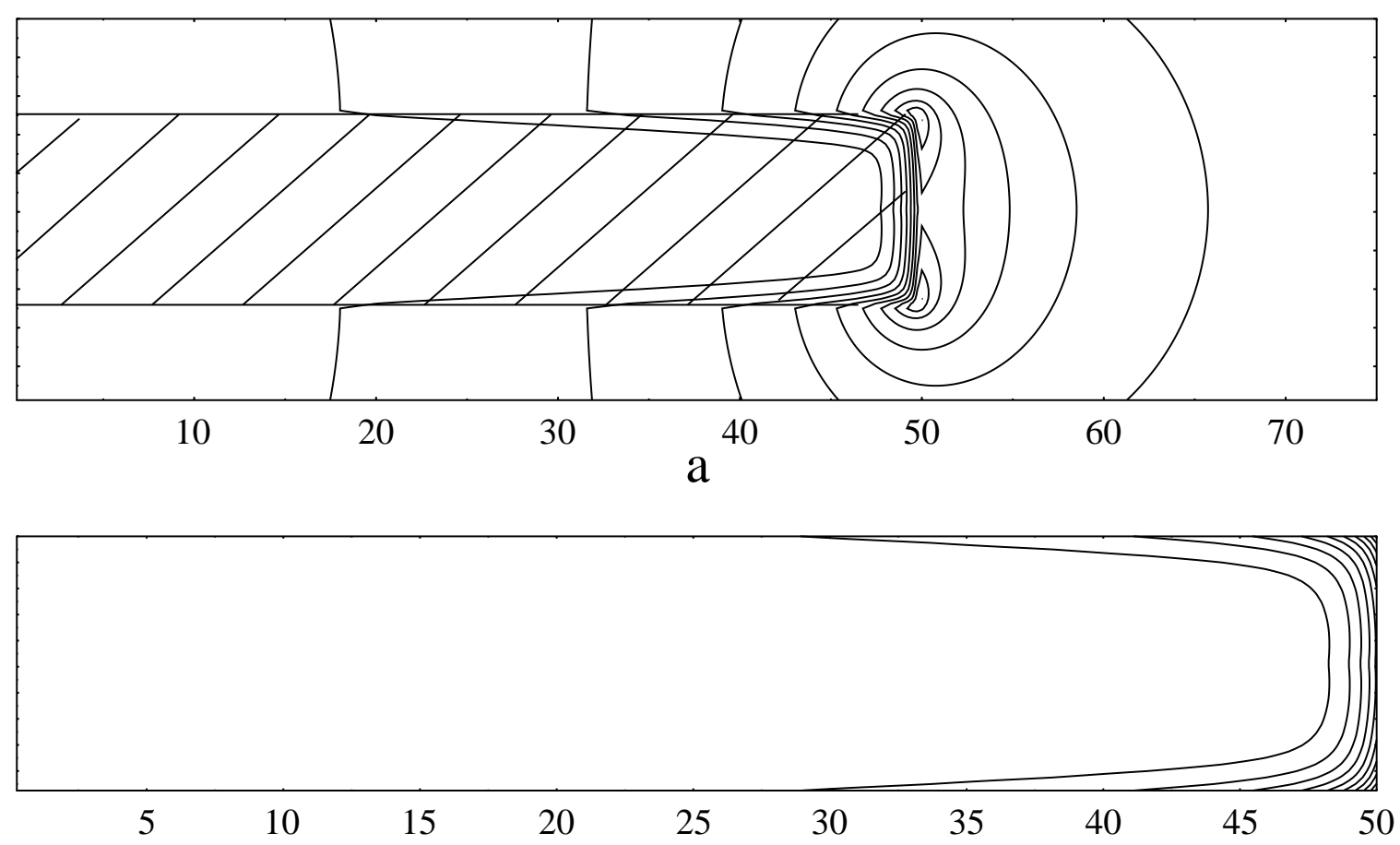

b
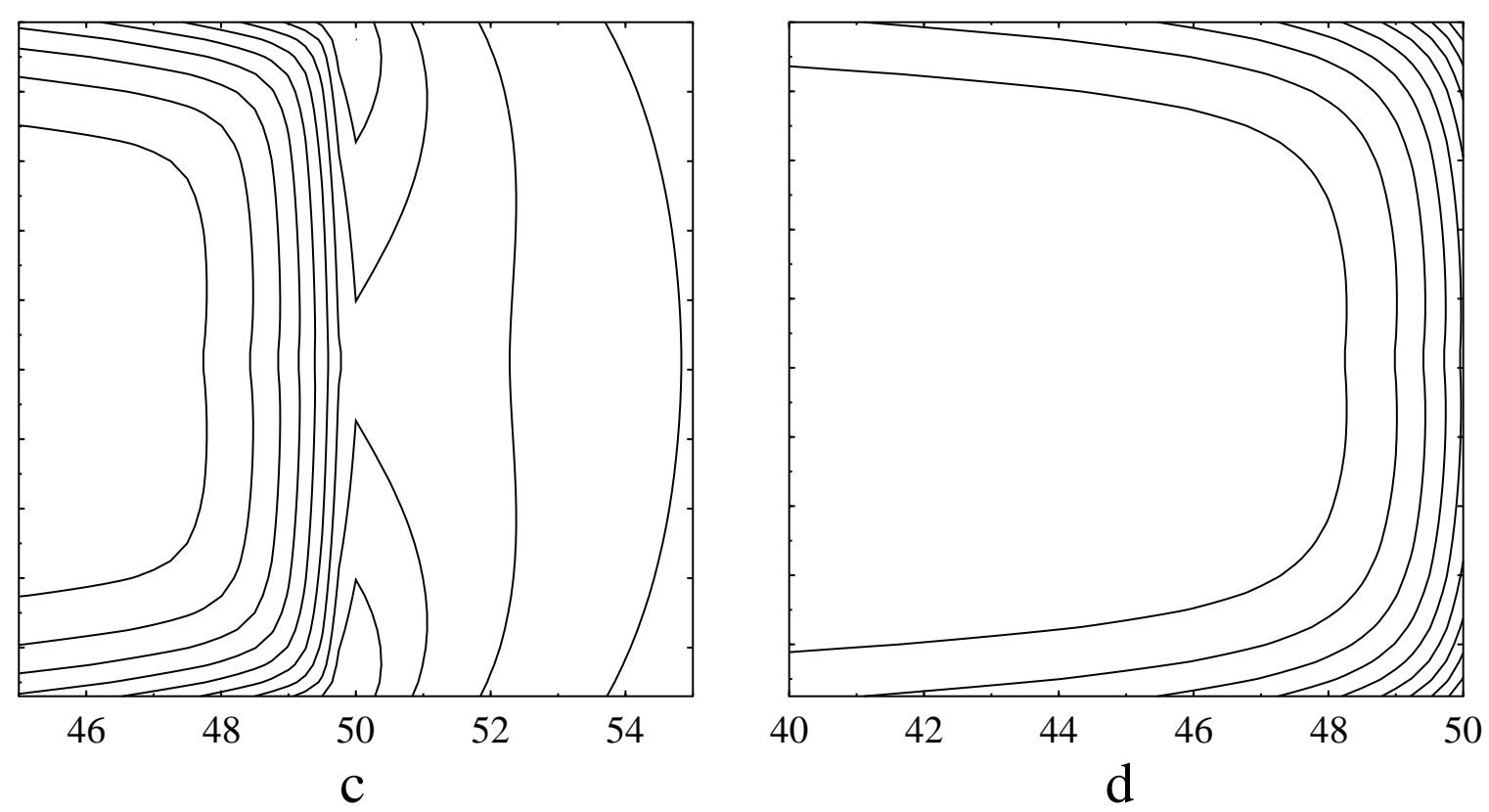

fig. 6 D.Yu.Vodolazov and I.L.Maksimov 


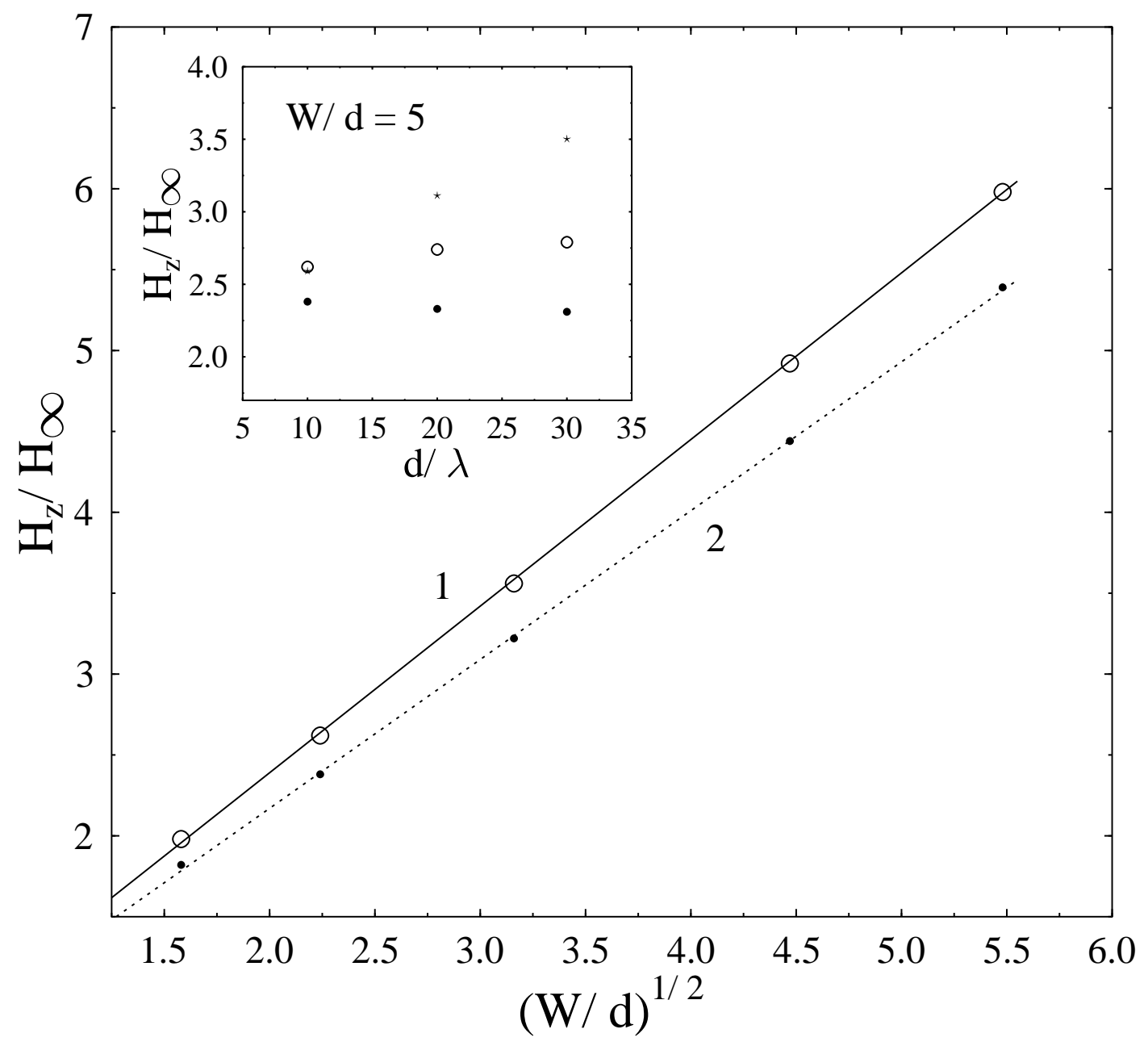

fig. 7 D.Yu.Vodolazov and I.L.Maksimov 


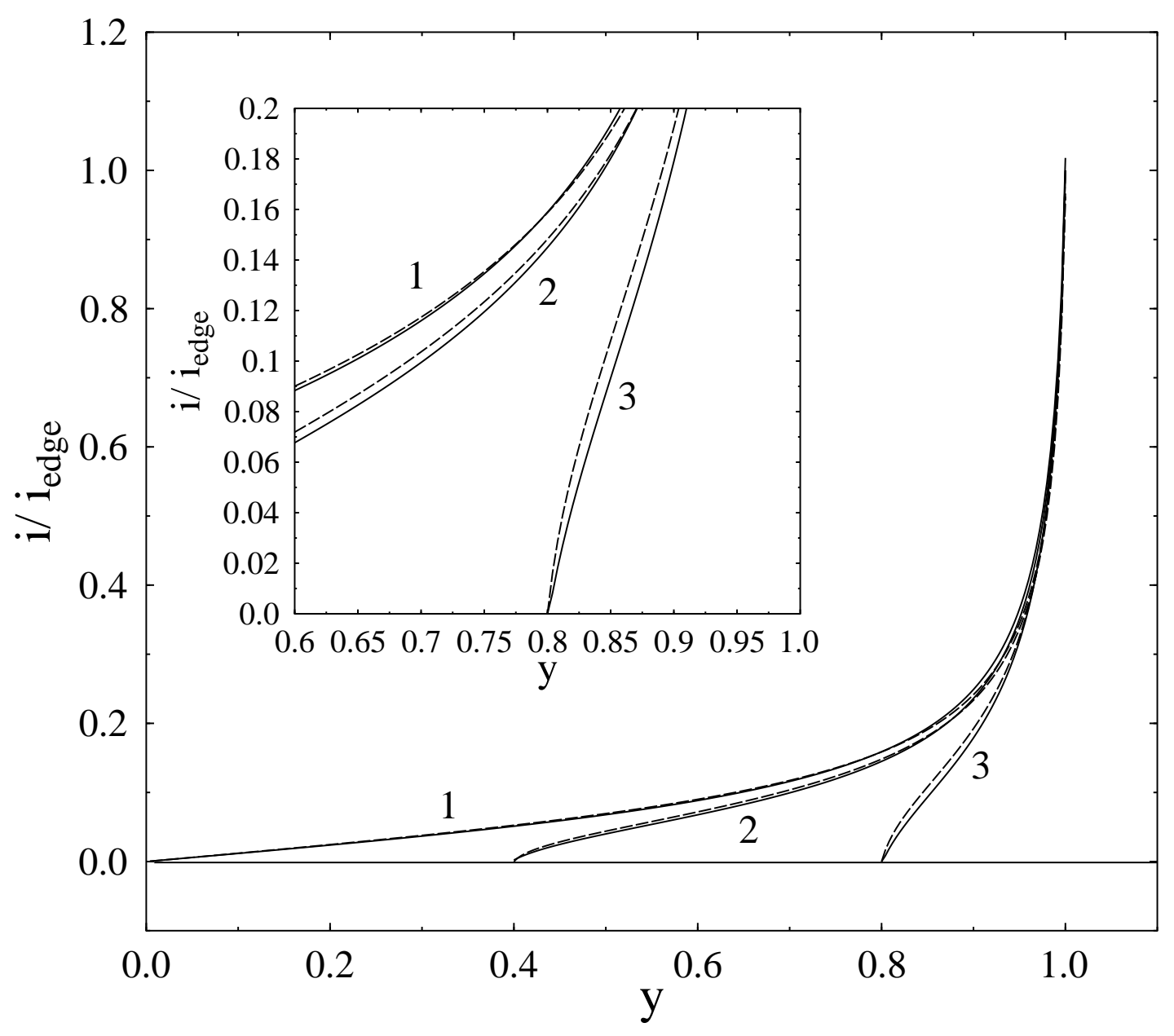

fig. 8 D.Yu.Vodolazov and I.L.Maksimov 


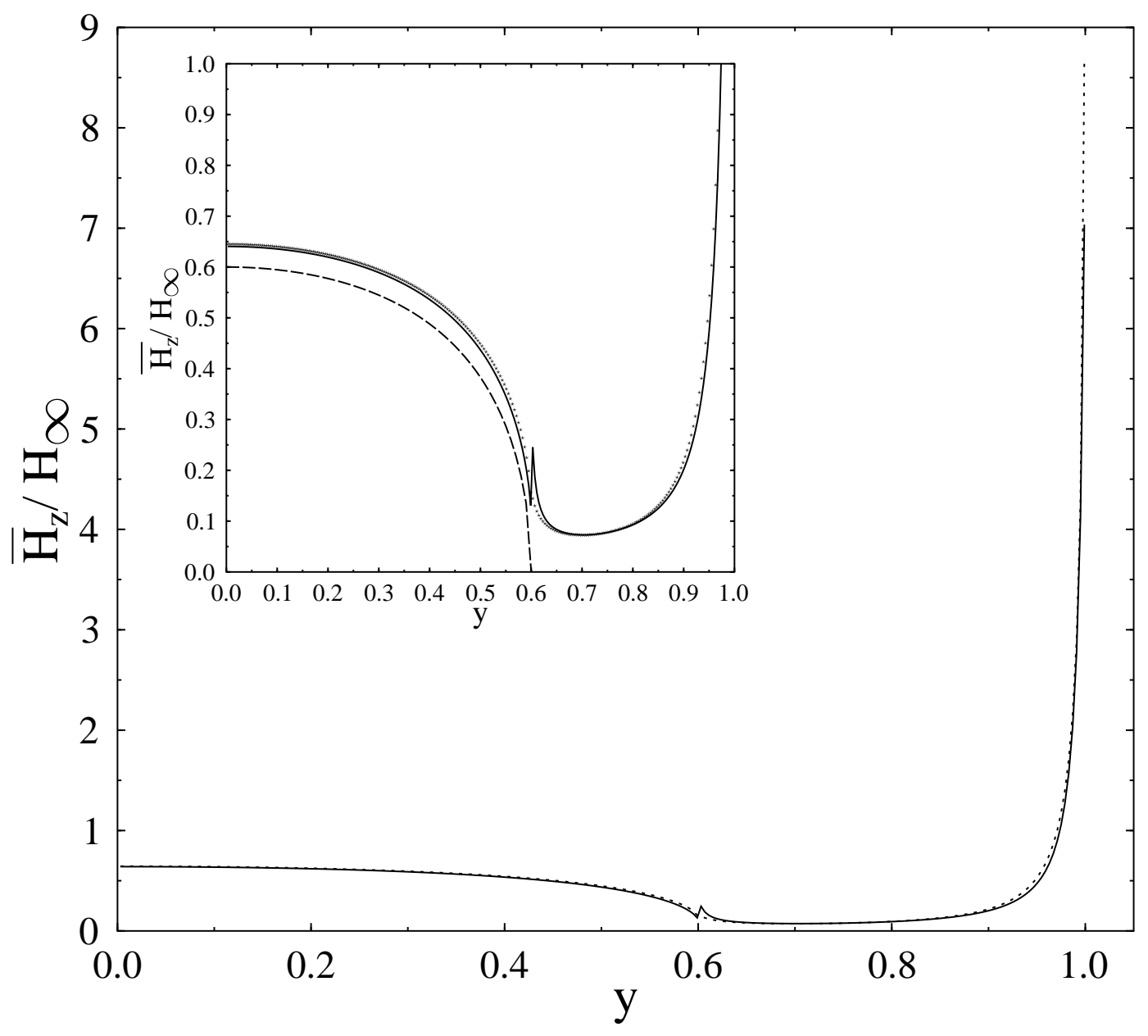

fig. 9 D.Yu.Vodolazov and I.L.Maksimov 


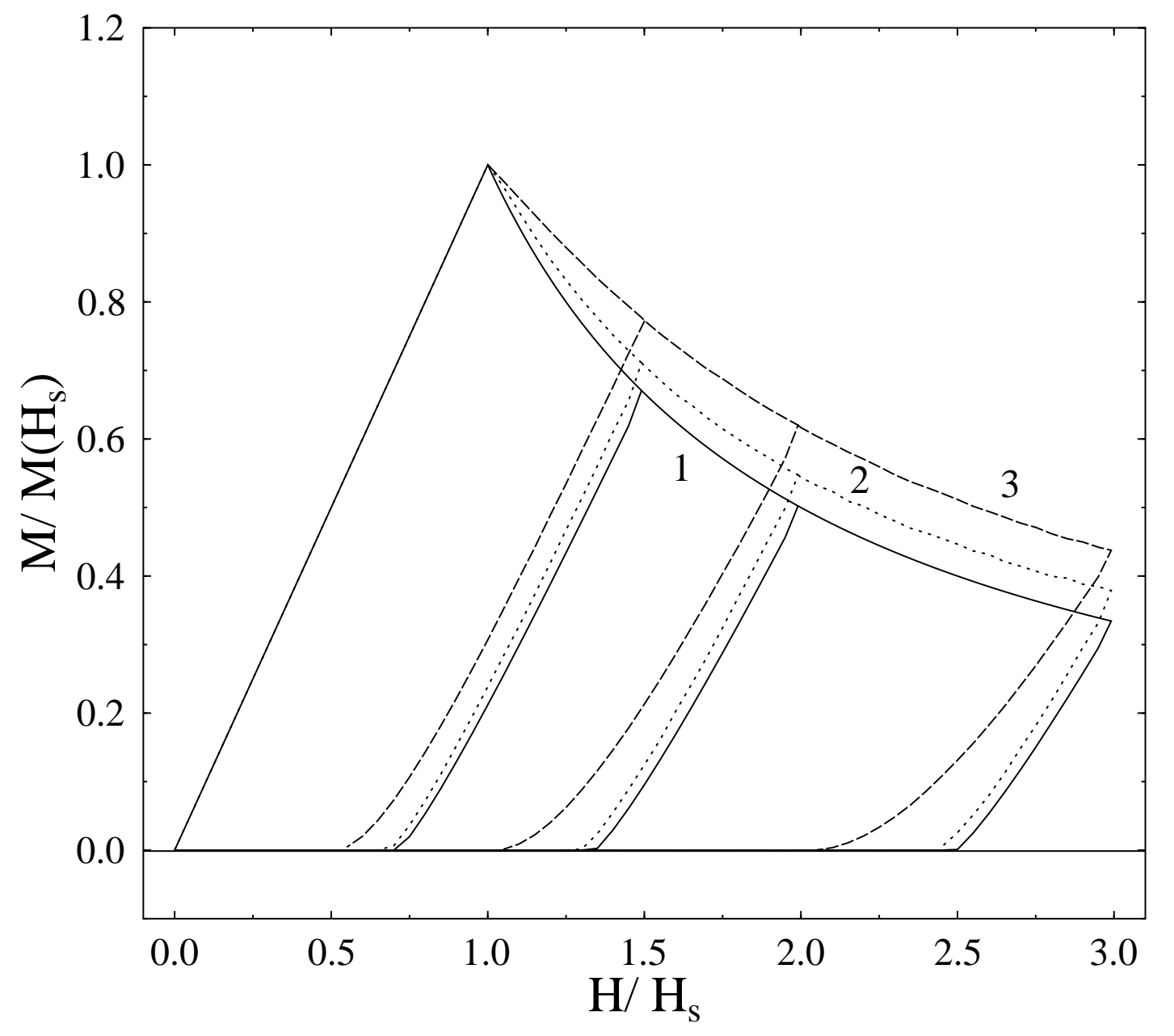

fig. 10 D.Yu.Vodolazov and I.L.Maksimov 